\title{
2018 Status on KIT Gyrotron Activities
}

$\underline{\text { John Jelonnek }}^{1,2}$, G. Aiello ${ }^{3}$, K. Avramidis ${ }^{1}$, G. Gantenbein ${ }^{1}$, G. Grossetti ${ }^{3}$, S. Illy ${ }^{1}$, Z. C. Ioannidis ${ }^{1}$, J. Jin ${ }^{1}$, P. Kalaria ${ }^{1}$, A. Marek ${ }^{1}$, I. Gr. Pagonakis ${ }^{1}$, T. Rzesnicki ${ }^{1}$, S. Ruess ${ }^{1,2}$,

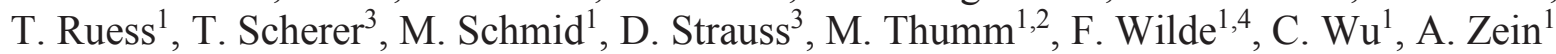

${ }^{1} \mathrm{IHM},{ }^{2} \mathrm{IHE},{ }^{3} \mathrm{IAM}$, Karlsruhe Institute of Technology (KIT), 76131 Karlsruhe, Germany

${ }^{4}$ Max-Planck-Institut für Plasmaphysik, Teilinstitut Greifswald, 17491 Greifswald, Germany

\section{Introduction}

Karlsruhe Institute of Technology (KIT) is focusing on the development of gyrotron oscillators and related components for Electron Cyclotron Resonance Heating (ECRH) and Current Drive (CD) systems for magnetically confined nuclear fusion plasmas. It includes the participation in the developments for W7-X [1], ITER [2], TCV [3] and a future EU DEMOnstration power plant (DEMO) [4]. In 2017, KIT has been invited by IAP to collaborate in a joint DFG-RSF project on fundamental research for future generation of ultrashort pulses in the millimeter and submillimeter wave bands for spectroscopy and diagnostics in various areas based on passive mode-locking in gyrodevices with nonlinear electron cyclotron absorber in the feedback loop. This presentation is summarizing the 2018 status on the KIT gyrotron activities.

\section{Status on the KIT fusion gyrotron developments}

The fig. 1 shows the major fusion gyrotrons in development at KIT. That are the W7-X $140 \mathrm{GHz}$ $1 \mathrm{MW}$ CW series gyrotron, the ITER $170 \mathrm{GHz} 1 \mathrm{MW}$ short-pulse (ms) prototype, and, finally, the KIT $170 \mathrm{GHz} 2 \mathrm{MW}$ short-pulse (ms) pre-prototype.

In February 2016 the full operation of the initial ECRH system of the stellarator W7-X started successfully. It is using 9 series gyrotrons produced by Thales Electron Devices (TED), France and 1 gyrotron manufactured by CPI, USA. In 2018, all 10 gyrotrons were operated and close to $8 \mathrm{MW}$ EC heating power was injected into the plasma already.

Since the successful start of W7-X, KIT has been focusing on the development of the first EU $170 \mathrm{GHz}$ $1 \mathrm{MW}$ hollow-cavity gyrotron for ITER. This development is coordinated by Fusion for Energy (F4E) and is ongoing within the European GYrotron Consortium (EGYC). The first industrial CW prototype bases on the $1 \mathrm{MW}$ short-pulse (SP) modular preprototype gyrotron that has been manufactured and tested at KIT earlier [5]. The experiments with the $\mathrm{CW}$ industrial prototype gyrotron are running in two phases. In 2016, the first phase was completed at KIT. Major results were an RF output power of above 0.9 MW in short-pulses. The measured Gaussian mode content of the RF output beam was higher than $97 \%$. Pulses with a duration of $180 \mathrm{~s}$ (limited by the max. possible pulse length of the high-voltage power supply at KIT) delivered an RF output power higher than $0.8 \mathrm{MW}$ with $38 \%$ efficiency (in depressed collector operation) [6]. The second phase of the experiments is currently ongoing at EPFL-SPC, Lausanne. In parallel, at KIT, the target is the optimization of the
RF output power and efficiency. Experiments with a modified SP modular pre-prototype are ongoing therefore. Various different schemes of depressed collector operation have been tested. The target is to achieve a total gyrotron efficiency higher than $50 \%$. Moreover, different designs of beam tunnels between electron gun and cavity are under consideration.

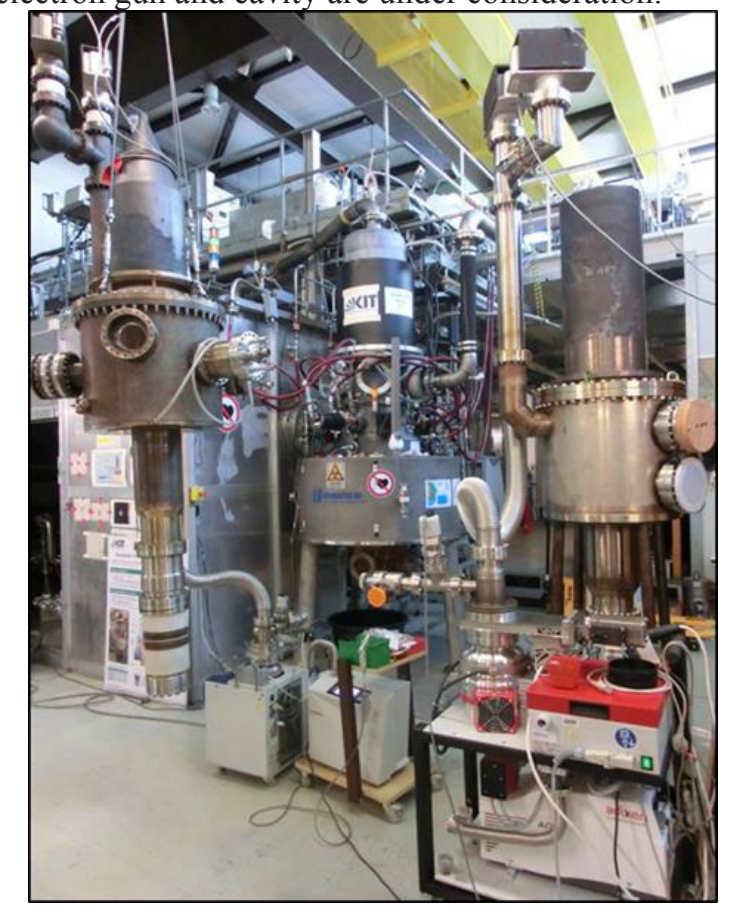

Fig. 1. The KIT fusion gyrotron family: KIT $170 \mathrm{GHz}$ $2 \mathrm{MW}$ short-pulse (ms) pre-prototype; W7-X $140 \mathrm{GHz}$ $1 \mathrm{MW}$ CW series gyrotron; ITER $170 \mathrm{GHz} 1 \mathrm{MW}$ shortpulse (ms) prototype (from left to right).

Based on the successful start of operation of the W7-X gyrotron, already in 2015, EPFL-SPC, Lausanne, Switzerland, invited KIT to collaborate in the physical design of a new TCV dual-frequency 84/126 $\mathrm{GHz}$ gyrotron with $1 \mathrm{MW}$ RF output power and $2 \mathrm{~s}$ pulse length. The boundary condition in this development was to keep the technical design as close as possible to the W7-X gyrotron development. Nevertheless, several improvements, e.g. a triode-type magnetron injection electron gun, have been introduced. Just before the Russian-German Workshop 2018, EPFL operated the new TCV gyrotron for the first time. The RF output beam shows a promising Gaussian mode content. Additionally based on the original W7-X design, and, in parallel to the TCV development, KIT was looking into different options for a possible $140 \mathrm{GHz} 1.5 \mathrm{MW}$ gyrotron upgrade for W7$\mathrm{X}$. A possible physical design based on an upgrade to the TE28,10 cavity mode were presented. 
In frame of the EUROfusion project, the basic research and development of a multi-megawatt gyrotron for future EU DEMO is ongoing. The $170 \mathrm{GHz}$, 2 MW short-pulse coaxial-cavity pre-prototype at KIT has already exhibited excellent performance in pulses of several ms [7]. The next step towards DEMO is to prove experimentally its capability for long-pulse operation. The coaxial-cavity pre-prototype was upgraded with new, water-cooled components and experiments targeting at $100 \mathrm{~ms}$ pulses are starting. To keep the development path towards the DEMO gyrotron as fast and cost-effective as possible, the design of a $2 \mathrm{MW}, 170 / 204 \mathrm{GHz}$ coaxial gyrotron has been initiated using the existing $170 \mathrm{GHz}$ coaxial-cavity gyrotron as a starting point.

The target of significant better than $60 \%$ efficiency for the DEMO gyrotron implies the development of advanced, Multi-Stage Depressed Collectors (MDCs). Given that in the gyrotron the electrons are facing a strong magnetic field at the collector region, the required separation of electrons according to their energy, necessary for MDC operation, is quite challenging. Investigations on different MDC concepts resulted in a configuration, which is based on the ExB drift concept [8] and adopts helical electrodes. The simulation results, carefully taking into account the influence of secondary electrons, demonstrate a gyrotron efficiency of higher than $63 \%$ with a two-stage collector.

To allow gyrotron frequency step-tunability the CVD-diamond Brewster-angle window is under research and development. A major challenge is to produce a large diamond disk of $180 \mathrm{~mm}$ diameter and $2 \mathrm{~mm}$ thickness. New manufacturing technologies are under investigation to increase the diameter up to the required value. Growth tests at the site of the industrial partner (Diamond Materials GmbH, Freiburg, Germany) were initiated with promising first results.

Vital to the future gyrotron $\mathrm{R} \& \mathrm{D}$ is the new Fusion Long Pulse Gyrotron Lab (FULGOR), a test stand able to support the development of up to $4 \mathrm{MW}$ continuous wave gyrotrons at frequencies up to $240 \mathrm{GHz}$ [9]. Within the EUROfusion project, the basic specification for a super-conducting $10.5 \mathrm{~T}$ cryogen-free magnet for FULGOR has been prepared. The magnet is expected to be delivered by mid-2019.

\section{Contributions to the Joint DFG-RSF Project - Generation of Ultra-Short Microwave Pulses -}

In a joined DFG-RSF project of IAP and IHM, the generation of a periodic sequence of powerful short RF pulses is studied [10]. Simulations for the design of a simple quasi-optical feedback system have been performed at KIT using the KIT code KARLESSS [11]. Full-wave PIC simulations including the interaction between electrons and electromagnetic waves are performed with the advanced simulation program PICLas [12], developed by the Institute of Aerodynamics and Gas Dynamics (IAG) at the University of Stuttgart. The required high-order hexahedral meshes are generated with the mesh-generator HOPR, also developed by the IAG.

\section{Acknowledgement}

This work is partly supported by Fusion for Energy under the Grants F4E-GRT-432 and F4E-GRT-553 to the European Gyrotron Consortium (EGYC), F4EOPE-458 to KIT and F4E-OPE-447 to TED. EGYC is a collaboration among SPC, Switzerland; KIT, Germany; HELLAS, Greece; IFP-CNR, Italy. Other parts of this work are partly carried out within the framework of the EUROfusion Consortium and are receiving funding from the Euratom research and training programme 2014-2018 under grant agreement No 633053. The views and opinions expressed herein do not necessarily reflect those of F4E and the European Commission. A part of this work was carried out using the HELIOS supercomputer system at Computational Simulation Centre of International Fusion Energy Research Centre (IFERC-CSC), Aomori, Japan, under the Broader Approach collaboration between Euratom and Japan, implemented by Fusion for Energy and QST. Part of the simulations was performed on the EUROfusion High Performance Computer (Marconi-Fusion). The joint IAP-IHM research on the generation of ultrashort pulses is ongoing in frame of the joint RSF-DFG project (Je 711/1-1).

\section{References}

1. Erckmann $V$. et al. ECRH and W7-X, an Intriguing Pair // AIP Proc. 20th Top. Conf. on RF-Power in Plasmas, Sorrento, Italy, P2.06 (2013).

2. Alberti $S$. et al. Dual-frequency, $126 / 84 \mathrm{GHz}, 1 \mathrm{MW}$ gyrotron for the upgrade of the TCV EC-system // Proc. IRMMW-THz Conf., Hong Kong (2015).

3. Darbos C. et al. Status of the ITER EC System // Proc. IEEE PPPS, San Francisco, USA, P5-22 (2013).

4. Tran M. Q. et al. EU DEMO Heating and Current Drive: Physics and Technology // Proc. 26th Fusion Energy Conf., Kyoto, FIP/7-7 (2016).

5. Rzesnicki T. et al. Recent experimental results of the European $1 \mathrm{MW}, 170 \mathrm{GHz}$ short-pulse gyrotron prototype for ITER // Proc. IRMMW-THz Conf., Hong Kong (2015).

6. Ioannidis $Z$. et al. First CW Experiments with the EU ITER $1 \mathrm{MW}, 170 \mathrm{GHz}$ Industrial Prototype Gyrotron // Proc. IEEE IVEC, London (2017).

7. Rzesnicki T. et al. $2 \mathrm{MW}, 170 \mathrm{GHz}$ Coaxial-Cavity Short-Pulse Gyrotron - Single Stage Depressed Collector Operation // Proc. IRMMW-THz Conf., pp. 1-2 (2014).

8. Pagonakis I. et al. A New concept for the collection of an electron beam configured by an externally applied axial magnetic field // IEEE Trans. Plasma Sci., vol. 36, no. 2, pp. 469-480 (2008).

9. Schmid M. et al. The 10 MW EPSM modulator and other key components for the KIT gyrotron test facility FULGOR // Fus. Eng. Des. (2017).

10. Ginzburg, N. S., et al. Generation of a periodic sequence of powerful ultrashort pulses in a traveling wave tube with bleachable absorber in the feedback loop." Technical Physics Letters, pp. 836-839 (2015).

11. Marek A. et al., Simulation of electromagnetic fields scattered from arbitrary shaped electric conductors // EPJ Web of Conferences 149, 04016 (2017).

12. Munz, C.-D. et al. Coupled particle-in-cell and direct simulation Monte Carlo method for simulating reactive plasma flows //Comptes Rendus Mécanique (2014). 